\title{
Factoring Multivariate Polynomials Over the Integers
}

\author{
By Paul S. Wang and Linda Preiss Rothschild*
}

\begin{abstract}
An algorithm for the irreducible factorization of any multivariate polynomial over the integers is given. It is much faster than the classical method ascribed to Kronecker. The algorithm begins by making substitutions for all but one of the variables with selected integers, giving a polynomial in just one variable. This univariate polynomial is then factored by a known method, which uses an algorithm of Berlekamp for factoring univariate polynomials over finite fields. The multivariate factors are constructed from the univariate ones by a kind of Hensel algorithm. The procedure has been implemented in the algebraic manipulation systems MACSYMA and SCRATCHPAD. A number of machine examples with timing are included.
\end{abstract}

1. Introduction. We describe an algorithm for the irreducible factorization of any multivariate polynomial over the rational integers, $\mathbf{Z}$. This algorithm makes use of Berlekamp's algorithm for factoring univariate polynomials modulo a prime number [1], [2]. Our algorithm is much faster than the classical method ascribed to Kronecker [10, pp. 135-136]. Experience of machine computation bears this out in both univariate and multivariate factoring with almost no exceptions.

Essentially, our algorithm first reduces a given multivariate polynomial to a polynomial in one variable by substituting integers for the other variables. The resulting univariate polynomial is then factored over $\mathbf{Z}$ by an algorithm which uses the Berlekamp algorithm with a small prime. The univariate factors over the integers are used in turn to construct the irreducible multivariate factors by using a variation of a kind of " $p$ adic" interpolation based on Hensel's lemma originally suggested by Zassenhaus [12].

The entire algorithm has been implemented in the LISP programming language [7] for the algebraic manipulation system MACSYMA [13] at Project MAC, Massachusetts Institute of Technology. It is also implemented in the SCRATCHPAD system at the Thomas J. Watson Research Center, IBM.

A list of polynomials factored by this computer program is included in an appendix. The running time for each problem is indicated. These timings are made on the MATHLAB time-sharing system at MAC which uses a PDP-10 computer with a memorycycle time of about two microseconds.

In his thesis, Musser [9] has described an algorithm for factoring a univariate polynomial over the integers in the SAC-1 system [4]. He has also discussed briefly some

Received August 3, 1972 ; revised March 4, 1974.

AMS (MOS) subject classifications (1970). Primary 10M05, 12-04, 12C05; Secondary 10D05, $12 \mathrm{E} 05$.

*Work reported herein was supported in part by Project MAC, an M.I.T. interdepartmental laboratory sponsored by the Advanced Research Projects Agency (ARPA), Department of Defense, under Office of Naval Research Contract N00014-70-A-0362-0001.

Copyright $\odot$ 1975, American Mathematical Society 
ideas of extending his algorithm to multivariate factorization. But no specific algorithms or programs have been given.

We are grateful to Stan Brown, Joel Moses, Richard Fateman, David Yun and Rich Schroeppel for assistance and suggestions. We would also like to thank G. E. Collins and D. Musser for making available to us an outline of their program for the Berlekamp algorithm. We also wish to thank the referee whose comments and suggestions were very helpful in revising this paper.

2. Preliminaries and an Outline. Polynomials over $Z$, in several variables, $x, x_{2}$, $x_{3}, \ldots, x_{n}$, form a unique factorization domain $Z\left[x, x_{2}, \ldots, x_{n}\right]$. Let $U\left(x, x_{2}, x_{3}, \ldots, x_{n}\right)$ be a multivariate polynomial in $\overline{\mathbf{Z}}\left[x, x_{2}, \ldots, x_{n}\right]$. By choosing a main variable, say $x$, we may write $U$ as a polynomial in $x$,

$$
U\left(x, x_{2}, \ldots, x_{n}\right)=U_{m} x^{m}+U_{m-1} x^{m-1}+\ldots+U_{1} x+U_{0}
$$

with coefficients $U_{i}$ in $\mathrm{Z}\left[x_{2}, \ldots, x_{n}\right], i=0,1, \ldots, m$. We may assume that the leading coefficient of $U, \operatorname{lc}(U)=U_{m}$, with respect to the main variable is not zero. Therefore, the degree of $U, \operatorname{deg}(U)$, is $m . \operatorname{CONT}(U)$, the content of $U$ with respect to the main variable, is defined as

$$
\operatorname{CONT}(U)=\operatorname{GCD}\left(U_{0}, U_{1}, \ldots, U_{m}\right),
$$

where GCD stands for the common divisor of greatest degree. The principal part of $U$, $\operatorname{pp}(U)$, is equal to $U / \operatorname{CONT}(U) . U$ is primitive if $\operatorname{CONT}(U)=1 . U$ is squarefree if $U$ has no repeated factors.

For any set, $F=\left\{f_{1}, f_{2}, \ldots, f_{r}\right\} \subset \mathrm{Z}\left[x_{2}, x_{3}, \ldots, x_{n}\right]$, the ideal generated by $F$, denoted by $\left(f_{1}, f_{2}, \ldots, f_{r}\right)$, is defined as the set,

$$
\left\{g_{1} f_{1}+g_{2} f_{2}+\ldots+g_{r} f_{r}: g_{i} \in \mathbf{Z}\left[x_{2}, \ldots, x_{n}\right] \forall i\right\}
$$

(see [10, Section 16]). The set $F$ need not be finite. For any integer, $k>0$ and any ideal $\mathfrak{8}, \mathfrak{8}^{k}$ denotes the ideal generated by all products of the form $h_{1} h_{2} \ldots h_{k}, h_{i} \in$ 8, $i=1,2, \ldots, k$.

If $A$ and $B$ are polynomials and $\delta$ is an ideal in $\mathrm{Z}\left[x, x_{2}, \ldots, x_{n}\right]$, we define $A \equiv B \bmod \&$ if $A-B \in$ 8, i.e., if $A-B$ is divisible by an element of 8 . For example, if $8=\left(x_{2}-a_{2}, x_{3}-a_{3}, \ldots, x_{n}-a_{n}\right)$ with $a_{2}, \ldots, a_{n}$ integers, $A\left(x, x_{2}, \ldots, x_{n}\right) \equiv$ $A\left(x, a_{2}, \ldots, a_{n}\right) \bmod$ for $A\left(x, a_{2}, \ldots, a_{n}\right)$ is the remainder of dividing $A$ by every $x_{i}-a_{i}, i=2, \ldots, n ; b^{k}$ is then the ideal generated by all polynomials of the form,

$$
\prod_{i=2}^{n}\left(x_{i}-a_{i}\right)^{c_{i}} \text { with } \sum_{i=2}^{n} c_{i}=k, c_{i} \geqslant 0
$$

For this ideal 8 we define, for any positive integer. $k$,

$$
A=B \bmod \boldsymbol{B}^{k} \quad \text { if } A \equiv B \bmod \boldsymbol{B}^{k} \text { and } \operatorname{deg}(A) \text { in } x_{2}, \ldots, x_{n}<k .
$$

Similarly, $A=B \bmod (q)$ for any prime power $q>2$ if $A \equiv B \bmod (q)$ and the coefficients of $A$ are between $-q / 2$ and $q / 2$.

Let us outline the five major steps in our algorithm for the irreducible factorization of $U$ over $\mathbf{Z}$. 
I. Primitive and Squarefree. If $U$ is not primitive, $\operatorname{CONT}(U)$ and $\operatorname{pp}(U)$ may be factored separately. Thus, we may assume that $U$ is primitive. Let

$$
\operatorname{GCD}\left(U\left(x, x_{2}, \ldots, x_{n}\right), \partial U / \partial x\right)=D .
$$

Note that $U$ has a repeated factor $G$ if and only if $G$ divides $D$ and $U / D$ is squarefree. The algorithm can be continued by factoring $D$ and $U / D$ separately.

II. Substitution. Find a set of integers, $\left\{a_{2}, a_{3}, \ldots, a_{n}\right\}$, (not necessarily distinct) such that $\tilde{U}(x)=U\left(x, a_{2}, \ldots, a_{n}\right)$ is squarefree and $\operatorname{deg}(\tilde{U})=\operatorname{deg}(U)$.

III. Univariate Factorization. Apply the factoring algorithm for a univariate polynomial to find a factorization

$$
\widetilde{U}(x)=\widetilde{p}_{1}(x) \ldots \widetilde{p}_{r}(x), \quad r \geqslant 1,
$$

over the integers. If $\tilde{U}(x)$ is irreducible, so is $U$ and the algorithm terminates. This process also produces a prime $q$ such that $\widetilde{U}(x)$ is squarefree modulo $q$ (see Section 5).

IV. Construction of Multivariate Factors.

(i) Coefficient Bound. Let $V=U\left(x, y_{2}+a_{2}, \ldots, y_{n}+a_{n}\right)$. Find a number $B$ such that for any integer coefficient $c$ of any divisor of $\operatorname{lc}(V) V, B>|c|$. Let $j$ be the smallest integer such that $2 B<q^{2 j}=b$. The prime power $b$ is used as a modulus in step (ii).

(ii) Construction of Factors. Equation (1) can be written as

$$
\widetilde{P}_{1}(x) \widetilde{P}_{2}(x) \ldots \widetilde{P}_{r}(x) \equiv U\left(x, \ldots, x_{n}\right) \bmod z
$$

where 8 is the ideal $\left(x_{2}-a_{2}, x_{3}-a_{3}, \ldots, x_{n}-a_{n}\right)$. An extended Zassenhaus algorithm based on a variant of Hensel's lemma (see Section 8 ) is used to compute, from the above congruence, relatively prime polynomials $P_{i}\left(x, x_{2}, \ldots, x_{n}\right), i=1, \ldots, r$, such that each $P_{i} \equiv \widetilde{P}_{i} \bmod \&$ and

$$
P_{1}\left(x, \ldots, x_{n}\right) \ldots P_{r}\left(x, \ldots, x_{n}\right) \equiv U\left(x, \ldots, x_{n}\right) \bmod \left(b, z^{h}\right)
$$

where $h=1+$ degree of $U$ in $x_{2}, \ldots, x_{n}$.

V. Finding Actual Factors. If $U\left(x, \ldots, x_{n}\right)$ is monic (with respect to the main variable $x)$, then any irreducible factor $G\left(x, \ldots, x_{n}\right)$ of $U$ over $\mathbf{Z}$ either is equal to some $P_{i}$ for $1 \leqslant i \leqslant r$ or is equal to the product of two or more $P_{i}$ 's $\bmod \left(b, b^{h}\right)$. If $U$ is not monic then these equivalences are up to the units in the coefficient domain of $U$ (see Section 9). In any event, the irreducible factors of $U$ are found from these $P_{i}$ 's by trial divisions.

3. A Complete Example. Now let us illustrate the whole algorithm by applying it to a specific multivariate polynomial, following the steps outlined in the previous section. The polynomial to be factored is

$$
\begin{aligned}
U(x, y, z)= & x^{4}+(-z+3) x^{3}+\left(z^{3}+(y-3) z-y^{2}-13\right) x^{2} \\
& +\left(-z^{4}+\left(y^{2}+3 y+15\right) z+6\right) x+y z^{4}+2 z^{3} \\
& +\left(-y^{3}-15 y\right) z-2 y^{2}-30
\end{aligned}
$$


I. $U$ is primitive since $\operatorname{lc}(U)=1$ (choosing $x$ as the main variable). $U$ is squarefree for $\operatorname{GCD}(U, \partial U / \partial x)=1$.

II. The substitution $y=0$ and $z=0$ is used because $\operatorname{deg}(U)=\operatorname{deg}(U(x, 0,0))$ and $U(x, 0,0)$ has no repeated factors. Thus, the ideal $z=(y, z)$ and

$$
\widetilde{U}(x)=U(x, 0,0)=x^{4}+3 x^{3}-13 x^{2}+6 x-30
$$

III. Factoring $\widetilde{U}(x)$ over $\mathbf{Z}$ (see Section 5), we have

$$
\widetilde{U}(x)=\left(x^{2}+2\right)\left(x^{2}+3 x-15\right) \text {. }
$$

IV. Starting from $\widetilde{U}(x) \equiv U(x, y, z) \bmod \delta$, we use the extended Zassenhaus algorithm to construct (see example in Section 8)

and

$$
\begin{array}{ll}
U \equiv\left(x^{2}-z x+2\right)\left(x^{2}+3 x-15\right) & \bmod 8^{2}, \\
U \equiv\left(x^{2}-z x+y z+2\right)\left(x^{2}+3 x-y^{2}-15\right) & \bmod 8^{3},
\end{array}
$$

$$
U \equiv\left(x^{2}-z x+y z+2\right)\left(x^{2}+3 x-y^{2}+z^{3}-15\right) \bmod z^{4}
$$

There is no need to go to a higher power of $\mathbf{z}$, because the last congruence is actually an equality over the integers.

$\mathrm{V}$. Trial division immediately gives the irreducible factorization of $U$ over $\mathbf{Z}$

$$
U(x, y, z)=\left(x^{2}-z x+y z+2\right)\left(x^{2}+3 x-y^{2}+z^{3}-15\right) .
$$

4. Selecting Integers for Substitution. The integers needed for substitution in step II exist. That is, one can show that if $U$ is squarefree, integers $a_{2}, a_{3}, \ldots, a_{n}$ can be chosen so that $\widetilde{U}(x)=U\left(x, a_{2}, \ldots, a_{n}\right)$ is still squarefree and $\operatorname{deg}(\widetilde{U})=\operatorname{deg}(U)$. The proof, which uses the discriminant, is omitted here. Suitable $a_{i}$ 's may be found by trial and error. The first choices for these $a_{i}$ 's should be 0,1 , and -1 for they usually make coefficients of $\widetilde{U}(x)$ small in size. It is desirable to use as many zeros as possible for the substitution, because each $a_{i}$ which is not zero can cause some intermediate expression growth when the extended Zassenhaus algorithm is applied in step IV.

For different substitutions, the number of factors, $r$, in (1) may be different. Since the larger $r$ is, the longer the entire algorithm takes, it is sometimes advantageous to try several substitutions and work with the one which gives minimum $r$. Our program does not attempt to do this not only because the number $r$ requires a fair amount of time to compute, but because a different substitution does not guarantee a smaller $r$.

The leading coefficient plays an important role in the factoring process. Factorization is easier if the leading coefficient is 1 ; for if $U$ is monic, then any factor of $U$ is monic. But if $U$ is not monic, then additional computation is required to determine the leading coefficient of each factor. Therefore, the main variable of $U$ is chosen so that $\operatorname{lc}(U)$ is 1 or small, in order to avoid or simplify later computations related to the leading coefficient. If several variables have a monic leading coefficient, it is best to choose the variable giving the smallest $\operatorname{deg}(\widetilde{U})$, thus limiting the number of possible factors. 
5. Factorization of Univariate Polynomials Over Z. In step III, an arbitrary squarefree polynomial $\widetilde{U}(x) \in \mathbf{Z}[x]$ is to be factored over $\mathbf{Z}$. The basic steps of this process are outlined here.

(a) Choose a prime $q$ such that $\widetilde{U}(x) \bmod (q)$ is squarefree and has the same degree as $\widetilde{U}(x)$. Let $u(x)=\widetilde{U}(x) \bmod (q)$.

(b) Factor $u(x)$ using Berlekamp's algorithm [1], [6] to obtain

$$
u(x) \equiv p_{1}(x) p_{2}(x) \ldots p_{t}(x) \bmod (q)
$$

with the $p_{i}$ 's distinct and irreducible over $\mathbf{Z}_{q}$. If $t=1, \tilde{U}$ is irreducible $\bmod (q)$, so $U$ is clearly irreducible over $\mathbf{Z}$, and the algorithm ends.

(c) Find an upper bound $\widetilde{B}$ on the magnitude of the coefficients of any possible factor of $\widetilde{U}(x)$, i.e. $\widetilde{B}$ is a number such that $\widetilde{B}>|c|$ for any coefficient $c$ of any factor of $\widetilde{U}$. Also find the least integer $d$ such that $q^{2^{d}} \geqslant 2 \operatorname{llc}(\widetilde{U}) \mid \widetilde{B}$.

(d) Use a " $p$-adic" algorithm by Zassenhaus [12] to construct $\hat{p}_{1}(x), \hat{p}_{2}(x), \ldots$, $\hat{p}_{t}(x)$ from (5) such that each $\hat{p}_{i} \equiv p_{i} \bmod (q)$ and

$$
\widetilde{U}(x) \equiv \hat{p}_{1}(x) \hat{p}_{2}(x) \ldots \hat{p}_{t}(x) \bmod \left(q^{2}\right) .
$$

(e) Use the algorithm TRUEFACTORS for the ideal $\left(q^{2^{d}}\right)$ to get a factorization of $\widetilde{U}(x)$ over $\mathbf{Z}$ (see Section 9).

$$
\widetilde{U}(x)=\widetilde{P}_{1}(x) \ldots \widetilde{P}_{r}(x), \quad 1 \leqslant r \leqslant t .
$$

If $r=1, U$ is irreducible over $\mathbf{Z}$. For example, we can carry out the above steps for the polynomial,

$$
\widetilde{U}(x)=x^{4}+3 x^{3}-13 x^{2}+6 x-30
$$

(a) $\widetilde{U}(x)$ is nonsquarefree mod (3). Choosing the next larger prime 5, we have

$$
u(x)=x^{4}-2 x^{3}+2 x^{2}+x \equiv \widetilde{U}(x) \bmod (5)
$$

which is squarefree.

(b) Factoring $u(x)$ over $Z_{5}$ gives

$$
u(x) \equiv x(x-2)\left(x^{2}+2\right) \bmod (5) \text {. }
$$

(c) If we take, for simplicity, $\widetilde{B}=300$, then $d=2$ since $5^{4}=625>2 \cdot 300$.

(d) Zassenhaus' algorithm described in Section 7 is applied to yield

$$
\widetilde{U}(x) \equiv(x+45)(x-42)\left(x^{2}+2\right) \bmod (625) \text {. }
$$

(e) Of the three factors above, only $\left(x^{2}+2\right)$ divides $\widetilde{U}(x)$ over $\mathbf{Z}$. Therefore, $\widetilde{U}(x)$ has only two irreducible factors. The other factor is

$$
x^{2}+3 x-15 \equiv(x+45)(x-42) \bmod (625) \text {. }
$$

An irreducible polynomial in $\mathbf{Z}[x]$ may have nontrivial factors modulo $q$. In fact, the number of factors, $t$, in step (b) depends on the prime $q$ chosen in step (a). Let $m=\operatorname{deg}(\widetilde{U})$ and $r$ be the number of irreducible factors of $\widetilde{U}(x)$ over Z. We see that 
$m \geqslant t \geqslant r$. In cases where $m$ is small, we may choose $q$ as the smallest prime that satisfies (a). If $m$ is rather large, a particular choice of $q$ may cause $t$ to be much greater than $r$. An extreme case is $r=1$ and $t=m$. To guard against such instances, one may want to try two or more primes, depending on the size of $m$, and use the one which gives minimum $t$.

According to Knuth [6], steps (a) and (b) take $O\left(\mathrm{~m}^{3}\right)$ units of time. Step (d) takes no more than $O\left(q \mathrm{tm}^{2}\right)$ units of time. For step (e) we use algorithm TRUEFACTORS which requires no more than $O\left(2^{t} \mathrm{~m}^{2}\right)$ units of time. In view of this exponential characteristic, it pays to make $(t-r)$ small. However, for each additional prime tried, the cost in time is approximately $O\left(\mathrm{~m}^{3}\right)$.

The referee informs us that a method due to $R$. Graham allows one to conclude irreducibility over $\mathbf{Z}$ from the way a polynomial factors modulo several primes. Currently, this method is not in our factoring algorithm.

6. Coefficient Bounds. There are two methods given in Knuth [6] for finding upper bounds on the magnitude of the coefficients of every possible factor of a given univariate polynomial $\widetilde{U}(x)$. One of these methods involves bounding the absolute value of the roots of $\widetilde{U}(x)$. Another uses matrix computations.

A coefficient bound which works in both the univariate and the multivariate cases is given by Gel'fond [5, p. 135]. Let the maximum coefficient magnitude and degrees of $U\left(x, x_{2}, \ldots, x_{n}\right)$ be $U_{\max }$ and $m, m_{2}, \ldots, m_{n}$, in the variables $x, x_{2}, \ldots$, $x_{n}$, respectively, then $U_{\max } e^{M}, M=m+m_{2}+\ldots+m_{n}$, bounas the magnitude of the coefficients of any divisor of $U$. We use this method to compute the upper bounds $\widetilde{B}$ (step (c)) and $B$ (step IV(i)). This is suggested to us by P. Weinberger.

The bounds computed are often much larger than they ought to be. If $\widetilde{B}$ is too large, then $d$, the number of iterations in the Zassenhaus algorithm, is too large. It is desirable to find a more accurate coefficient bound than the one we use here. MACSYMA enables the user to save computation time by electing to use a heuristic bound in the factoring process:

$$
\widetilde{B}=m \cdot \operatorname{MAX}\left(m,\left|\widetilde{U}_{0}\right|,\left|\widetilde{U}_{1}\right|, \ldots,\left|\widetilde{U}_{m}\right|\right) \cdot 2^{\{m / 2\}}, \quad m=\operatorname{deg}(\widetilde{U}),
$$

where the $\widetilde{U}_{i}$ 's are the coefficients of $\tilde{U}(x)$ and $\{m / 2\}$ stands for the least integer $\geqslant$ $m / 2$. This heuristic bound is sometimes still rather large. Arithmetic involving integers more than single precision (35 bits on a PDP-10) is handled by MACSYMA's arbitrary precision integer arithmetic routines.

It is conceivable, although not probable, for the heuristic $\widetilde{B}$ to be too small. In such a case, factors found by the algorithm may be reducible. Thus, one should be careful in electing to use the heuristic coefficient bound.

7. Zassenhaus' Algorithm for Factoring $\bmod q^{2 j}$. In this section, we describe how (6) is constructed from (5) in step (d) of Section 5. The algorithm is due to Zassenhaus [12] and is based on Hensel's lemma. Let us write (5) as

$$
\widetilde{U}(x) \equiv p_{10} p_{20} \ldots p_{t 0} \bmod (q)
$$


with $p_{i 0}$ 's distinct and irreducible over the field $\mathbf{Z}_{q}$. From these factors, the algorithm constructs $p_{i j}$, proceeding from $j$ to $j+1$, starting from $j=0$, such that

$$
\widetilde{U}(x) \equiv p_{1 j} p_{2 j} \ldots p_{t j} \bmod \left(q^{2 j}\right) \text { and } p_{i j} \equiv p_{i 0} \bmod (q) .
$$

It suffices to have such an algorithm for two relatively prime factors of $\widetilde{U}$, which can then be used recursively when $t>2$.

Let $F_{J}$ and $G_{0}$ be relatively prime polynomials in $\mathbf{Z}_{q}[x]$ such that $\operatorname{deg}\left(F_{0}\right)=$ $f>0, \operatorname{deg}\left(\mathcal{G}_{0}\right)=g>0, \operatorname{deg}(\tilde{U})=f+g$ and $\tilde{U}(x) \equiv F_{0}(x) G_{0}(x) \bmod (q)$.

Lemma. Polynomials $F_{j}, G_{j}, \alpha_{j}$ and $\beta_{j}, j \geqslant 0$, can be found such that $\operatorname{deg}\left(F_{j}\right)=$ $f, \operatorname{deg}\left(G_{j}\right)=g$,

$$
\widetilde{U}(x) \equiv F_{j} G_{j} \quad \bmod \left(q^{2^{j}}\right), \quad F_{j} \equiv F_{0}, \quad G_{j} \equiv G_{0} \quad \bmod (q)
$$

and

$$
\alpha_{j} F_{j}+\beta_{j} G_{j} \equiv 1 \bmod \left(q^{2 j}\right)
$$

with $\operatorname{deg}\left(\alpha_{j}\right)<g$ and $\operatorname{deg}\left(\beta_{j}\right) \leqslant f$. Furthermore, $F_{j}$ and $G_{j}$ are unique up to units. (Note that $\widetilde{U}(x)$ need not be monic.)

Proof. The lemma is true for $j=0$, because $\alpha_{0}$ and $\beta_{0}$ can be found uniquely by a polynomial remainder sequence method given in [6]. Let us assume that the lemma is true for $j=k-1$. We compute $C(x)$ by

$$
C(x)=\left(F_{k-1} G_{k-1}-\tilde{U}\right) / q^{2^{k-1}} \bmod \left(q^{2^{k-1}}\right) .
$$

Let $R_{1}(x)$ be the remainder of $\alpha_{k-1} C(x)$ divided by $G_{k-1}$, i.e., compute $R_{1}$ by

$$
R_{1}=\alpha_{k-1} C-G_{k-1} P \bmod \left(q^{2^{k-1}}\right)
$$

with $P(x)$ the quotient. Compute $R_{2}(x)$ by

$$
R_{2}=F_{k-1} P+\beta_{k-1} C \bmod \left(q^{2^{k-1}}\right) \text {. }
$$

It is easy to deduce that $\operatorname{deg}\left(R_{1}\right)<g, \operatorname{deg}\left(R_{2}\right) \leqslant f$ and, from (9),

$$
R_{1} F_{k-1}+R_{2} G_{k-1} \equiv C(x) \bmod \left(q^{2^{k-1}}\right) \text {. }
$$

Thus, if we compute $F_{k}(x)$ and $G_{k}(x)$ by

$$
F_{k}=F_{k-1}-q^{2^{k-1}} R_{2} \text { and } G_{k}=G_{k-1}-q^{2^{k-1}} R_{1},
$$

It follows from (10) and (11) that $F_{k}$ and $G_{k}$ satisfy (8) for $j=k$. Now compute $D(x)$ by

$$
D(x)=\left(\alpha_{k-1} F_{k}+\beta_{k-1} G_{k}-1\right) / q^{2^{k-1}} \bmod \left(q^{2^{k}}\right) .
$$

As before, we can find $S_{1}(x)$ and $S_{2}(x)$ such that $\operatorname{deg}\left(S_{1}\right)<g, \operatorname{deg}\left(S_{2}\right) \leqslant f$ and $S_{1} F_{k-1}+S_{2} G_{k-1} \equiv D(x) \bmod \left(q^{2}{ }^{k-1}\right)$. Therefore, if we compute $\alpha_{k}$ and $\beta_{k}$ by

$$
\alpha_{k}=\alpha_{k-1}-q^{2^{k-1}} S_{1} \text { and } \beta_{k}=\beta_{k-1}-q^{2^{k-1}} S_{2} \bmod \left(q^{2^{k}}\right),
$$

then (9) is satisfied with $j=k$. 
Now we show that $F_{k}$ and $G_{k}$ are unique up to units. Let us assume

$$
\widetilde{U}(x) \equiv F_{k}(x) G_{k}(x) \equiv A(x) B(x) \bmod \left(q^{2 k}\right) ;
$$

and, by induction,

$$
F_{k} \equiv F_{k-1} \equiv a A \quad \text { and } \quad G_{k} \equiv G_{k-1} \equiv a^{-1} B \bmod \left(q^{2^{k-1}}\right)
$$

for some unit $a$ in $Z /\left(q^{2^{k-1}}\right)$. That is,

$$
\begin{aligned}
& F_{k} \equiv a A+q^{2^{k-1}} C_{1}(x), \\
& G_{k} \equiv a^{-1} B+q^{2^{k-1}} C_{2}(x) \bmod \left(q^{2^{k}}\right)
\end{aligned}
$$

for some $C_{1}$ and $C_{2}, \operatorname{deg}\left(C_{1}\right) \leqslant \operatorname{deg}(A)$ and $\operatorname{deg}\left(C_{2}\right) \leqslant \operatorname{deg}(B)$. From (13) we have

$$
a A C_{2}+a^{-1} B C_{1} \equiv 0 \bmod \left(q^{2^{k-1}}\right) \text {. }
$$

$A$ and $B$ being relatively prime implies

$$
C_{1}=e_{1} A \text { and } C_{2}=e_{2} B \bmod \left(q^{2^{k-1}}\right)
$$

for $e_{1}$ and $e_{2}$ units in $\mathrm{Z} /\left(q^{2^{k-1}}\right)$. Hence, $A$ and $B$ are unit multiples of $F_{k}$ and $G_{k}$, respectively.

Therefore, (7) can be constructed and it is unique up to units in the ring $Z /\left(q^{2 j}\right)$.

Example. Let

$$
\widetilde{U}(x)=x^{4}+3 x^{3}-13 x^{2}+6 x-30
$$

Extend the following congruence to modulo 25:

$$
\widetilde{U}(x) \equiv\left(x^{2}-2 x\right)\left(x^{2}+2\right) \bmod (5) \text {. }
$$

Let $F_{0}=x^{2}-2 x, G_{0}=x^{2}+2$; we have

$$
F_{0} G_{0}-\tilde{U}(x)=5\left(-x^{3}+3 x^{2}-2 x+6\right)=5 C(x)
$$

$\alpha_{0}(x)=0$ and $\beta_{0}(x)=-(x+2)$ give

$$
\alpha_{0} F_{0}+\beta_{0} G_{0} \equiv C(x) \bmod (5) \text {. }
$$

Therefore, according to Eqs. (11) and (12),

$$
\begin{aligned}
& F_{1}(x)=F_{0}(x)-5(-x-2)=x^{2}+3 x+10 \\
& G_{1}(x)=G_{0}(x) \text { and } F_{1} G_{1} \equiv \tilde{U}(x) \bmod (25)
\end{aligned}
$$

8. An Extended Zassenhaus Algorithm. This algorithm computes (2) in step IV from (1) in step III. For the same reason as given in Section 7, we shall give the algorithm for two relatively prime factors. Let $F(x)$ and $G(x)$ be two relatively prime polynomials such that $F(x) G(x)=\widetilde{U}(x)$ over Z. It follows that

$$
U\left(x, x_{2}, \ldots, x_{n}\right) \equiv F(x) G(x) \bmod 8
$$


where 8 is the ideal $\left(x_{2}-a_{2}, x_{3}-a_{3}, \ldots, x_{n}-a_{n}\right)$. From $F$ and $G$ this algorithm constructs, proceeding from $k$ to $k+1$ starting from $k=1$, multivariate polynomials $F_{k}$ and $G_{k}$, with $F_{1}=F$ and $G_{1}=G$, such that

$$
F_{k}\left(x, x_{2}, \ldots, x_{n}\right) \equiv F(x) \quad \bmod \not, \quad G_{k}\left(x, x_{2}, \ldots, x_{n}\right) \equiv G(x) \bmod \&
$$

and

$$
U\left(x, x_{2}, \ldots, x_{n}\right) \equiv F_{k} G_{k} \bmod g^{k} .
$$

We shall first show how to get to $k=2$. Then, the general case is given by induction. The algorithm ends when $k$ reaches $h$ which equals $1+$ degree of $U$ in $x_{2}$, $\ldots, x_{n}$.

$$
\begin{aligned}
& \text { Let } y_{i}=x_{i}-a_{i}, i=2,3, \ldots, n \text {, and } \\
& \qquad V\left(x, y_{2}, \ldots, y_{n}\right)=U\left(x, y_{2}+a_{2}, \ldots, y_{n}+a_{n}\right) .
\end{aligned}
$$

We compute $R_{1}$ and $W_{1}$ by

and

$$
R_{1}\left(x, y_{2}, \ldots, y_{n}\right)=F(x) G(x)-V\left(x, y_{2}, \ldots, y_{n}\right)
$$

$$
W_{1}\left(x, y_{2}, \ldots, y_{n}\right)=R_{1} \bmod \dot{s}^{2} .
$$

Since is now the ideal $\left(y_{2}, \ldots, y_{n}\right)$, computations mod $8^{i}$ are done simply by dropping all the terms of degree greater than or equal to $i$ in $y_{2}, \ldots, y_{n}$, e.g., $y_{2} y_{3} \equiv 0$ $\bmod \mathbf{s}^{2}$.

Since $F(x)$ and $G(x)$ are relative prime, unique $\alpha_{i}(x)$ and $\beta_{i}(x)$ can be computed such that for all $i \leqslant m$

$$
\alpha_{i}(x) F(x)+\beta_{i}(x) G(x)=x^{i}
$$

with $\operatorname{deg}\left(\alpha_{i}\right)<\operatorname{deg}(G)$ and $\operatorname{deg}\left(\beta_{i}\right) \leqslant \operatorname{deg}(F)$. These $\alpha_{i}$ and $\beta_{i}$ are computed as the need arises and are stored for future use. This scheme is used at the suggestion of $J$. Moses.

For any polynomial $T\left(x, y_{2}, \ldots, y_{n}\right)$, let us denote by $\mathbf{A}(T)$ the polynomial obtained by substituting $\alpha_{i}(x)$ for $x^{i}$ in $T$ and by $\mathrm{B}(T)$ the polynomial obtained by substituting $\beta_{i}(x)$ for $x^{i}$ in $T$. Now compuce $F_{2}, G_{2}$ and $R_{2}$ by

$$
F_{2}\left(x, y_{2}, \ldots, y_{n}\right)=F(x)-\mathbf{B}\left(W_{1}\right), \quad G_{2}\left(x, y_{2}, \ldots, y_{n}\right)=G(x)-\mathbf{A}\left(W_{1}\right),
$$

and

$$
R_{2}=R_{1}+\mathbf{A}\left(W_{1}\right) \mathbf{B}\left(W_{1}\right)-\mathbf{A}\left(W_{1}\right) F-\mathbf{B}\left(W_{1}\right) G
$$

It is readily verified that

$$
\begin{gathered}
\mathbf{A}\left(W_{1}\right) F+\mathbf{B}\left(W_{1}\right) G=W_{1}, \quad \mathbf{A}\left(W_{1}\right) \mathbf{B}\left(W_{1}\right) \equiv 0 \bmod b^{2}, \\
V \equiv F_{2} G_{2} \quad \bmod 8^{2} \quad \text { and } \quad R_{2}=F_{2} G_{2}-V .
\end{gathered}
$$

Suppose we have, by induction, $F_{k-1}, G_{k-1}$ and $R_{k-1}$ such that, for $k \geqslant 2$, $V \equiv F_{k-1} G_{k-1} \quad \bmod 8^{k-1}, \quad F_{k-1} \equiv F \bmod 8, \quad G_{k-1} \equiv G \bmod 8$ and 


$$
R_{k-1}=F_{k-1} G_{k-1}-V
$$

Now let us compute $W_{k-1}, F_{k}, G_{k}$ and $R_{k}$ by

$$
\begin{gathered}
W_{k-1}=R_{k-1} \quad \bmod 8^{k}, \quad F_{k}=F_{k-1}-\mathbf{B}\left(W_{k-1}\right), \\
G_{k}=G_{k-1}-\mathbf{A}\left(W_{k-1}\right)
\end{gathered}
$$

and

$$
R_{k}=R_{k-1}+\mathrm{A}\left(W_{k-1}\right) \mathrm{B}\left(W_{k-1}\right)-\mathrm{A}\left(W_{k-1}\right) F_{k-1}-\mathrm{B}\left(W_{k-1}\right) G_{k-1} .
$$

Then, it can be deduced that

$$
F_{k} \equiv F \quad \bmod 8, \quad G_{k} \equiv G \bmod 8
$$

and

$$
F_{k} G_{k}-V \equiv R_{k} \equiv 0 \bmod \dot{8}^{k}
$$

Consequently, the congruence

$$
V\left(x, y_{2}, \ldots, y_{n}\right) \equiv F_{k}\left(x, y_{2}, \ldots, y_{n}\right) G_{k}\left(x, y_{2}, \ldots, y_{n}\right) \bmod 8^{k}
$$

can be constructed for any $k \geqslant 2$. The algorithm ends whenever $R_{k}$ is zero or when $k$ reaches $h$. It can be shown that $F_{k}$ and $G_{k}$ are unique up to units. Therefore, the $P_{i}$ 's in (2) are also unique up to units.

Integer arithmetic would be sufficient for this algorithm if it were not for the $\alpha_{i}(x)$ and $\beta_{i}(x)$ needed in (15) which normally have rational coefficients. To avoid the costly process of using rational arithmetic for the entire extended Zassenhaus algorithm, we can use a large enough modulus for our computation.

Let $B$ be an upper bound on the magnitude of the coefficients of any possible factor of $V\left(x, y_{2}, \ldots, y_{n}\right)$ or $\tilde{U}(x)$. We can use the prime $q$ chosen in Section 5 to form a prime power $b=q^{2 j}$ such that $b>2 \operatorname{llc}(U) \mid B$. It is easy to see that all computation can be done modulo $b$. However, a true bound is usually too large. In MACSYMA we provide an optional heuristic bound:

$$
B=\operatorname{MAX}\left(\widetilde{B}, h \cdot \operatorname{MAX}\left(h, v_{\max }\right) \cdot 2^{\{h / 2\}}\right)
$$

where $v_{\max }$ is the absolute value of the largest integer coefficient of $V$.

The computing time of this algorithm is dominated by the multiplication of multivariate polynomials in Eq. (17). If $V$ has $v$ terms, Eq. (17) takes no more than $v^{2}$ units of time. Thus, the entire algorithm takes $O\left(h v^{2}\right)$ units of time. And to construct the congruence (2) from (1), we need to apply this algorithm $r$ times.

Example. Given

$$
\begin{aligned}
U(x, y, z)= & x^{4}+(-z+3) x^{3}+\left((y-3) z-y^{2}-13\right) x^{2} \\
& +\left(\left(y^{2}+3 y+15\right) z+6\right) x \\
& +\left(-y^{3}-15 y\right) z-2 y^{2}-30 \\
F(x)= & \left(x^{2}+2\right), \quad G(x)=\left(x^{2}+3 x-15\right)
\end{aligned}
$$


and

$$
U(x, 0,0)=F(x) G(x)
$$

find $F_{2}, G_{2}, F_{3}$ and $G_{3}$.

To avoid rational arithmetic, we use 625 as a modulus; that is, all arithmetic will be done modulo 625. Let 8 be the ideal $(y, z)$; then $U \equiv F(x) G(x) \bmod 8$. Now

$$
W_{1}(x, y, z)=z\left(x^{3}+3 x^{2}-15 x\right)=F \cdot G-U \bmod 8^{2} .
$$

And we have

$$
\begin{array}{ll}
\alpha_{0}=-171 x-232, & \alpha_{1}=281 x-65, \\
\alpha_{2}=-283 x-160, & \alpha_{3}=64 x+130, \\
\beta_{0}=171 x-281, & \beta_{1}=281 x+283, \\
\beta_{2}=283 x-63, & \beta_{3}=-63 x+59,
\end{array}
$$

satisfying Eq. (15). All these $\alpha_{i}$ 's and $\beta_{i}$ 's are derived, as indicated in Section 7, from the relation $-(x+2) F+(x-1) G \equiv 1 \bmod (5)$ which can be obtained by a polynomial remainder sequence method.

We now can compute $\mathbf{A}\left(W_{1}\right)=0$ and $\mathbf{B}\left(W_{1}\right)=z x$ which give $F_{2}=x^{2}-z x+2$, $G_{2}=G$. Similarly, to find $F_{3}$ and $G_{3}$, we compute

$$
\begin{gathered}
W_{2}=-\left(y z-y^{2}\right) x^{2}-3 y z x+15 y z+2 y^{2}=R_{2} \bmod 8^{\circ}, \\
\mathbf{A}\left(W_{2}\right)=y^{2} \text { and } \mathbf{B}\left(W_{2}\right)=-y z
\end{gathered}
$$

This means that

$$
F_{3}=x^{2}-z x+y z+2 \text { and } G_{3}=x^{2}+3 x-y^{2}-15 .
$$

One can show that $F_{2} G_{2} \equiv U \bmod 8^{2}$ and $F_{3} G_{3} \equiv U \bmod 8^{3}$.

9. Obtaining True Factors. In this section, we describe the algorithm for obtaining actual factors of $U\left(x, x_{2}, \ldots, x_{n}\right)$ over the integers from the factorization

$$
U\left(x, \ldots, x_{n}\right) \equiv P_{1}\left(x, \ldots, x_{n}\right) \ldots P_{r}\left(x, \ldots, x_{n}\right) \bmod \left(b, 8^{h}\right)
$$

with $r \geqslant 2$, the $P_{i}$ 's distinct and irreducible.

The factorization (2) is unique up to units in the quotient ring $R=$ $\mathrm{Z}\left[x_{2}, \ldots, x_{n}\right] /\left(b, 8^{h}\right)$, though $R$ is not a unique factorization domain.

If $U$ is monic, then the $P_{i}$ 's are all monic and any irreducible factor $G\left(x, \ldots, x_{n}\right)$ of $U$ either is equal to some $P_{i}, 1 \leqslant i \leqslant r$, or is equal to the product of two or more $P_{i}$ 's $\bmod \left(b, b^{h}\right)$. If $U$ is not monic, then these equivalences are up to units in $R$. Suppose an irreducible factor $G$, of $U$ over $Z$, is equal to $H$ up to units in $R$. Then

$$
H^{*}=\operatorname{lc}(U) \operatorname{lc}(H)^{-1} H \equiv \operatorname{lc}(U) \operatorname{lc}(G)^{-1} G \quad \bmod \left(b, 8^{h}\right)
$$

and $G=\operatorname{pp}\left(H^{*}\right)$ taken over the integers. The quantity $\operatorname{lc}(U) \operatorname{lc}(H)^{-1}$ is easily computed. For example, for any $1 \leqslant i \leqslant r$, 


$$
\operatorname{lc}(U) \operatorname{lc}\left(P_{i}\right)^{-1}=\prod_{j=1 ; j \neq i}^{r} \operatorname{lc}\left(P_{j}\right) \bmod \left(b, s^{h}\right) .
$$

Evidently, if $H^{*}$ divides $\operatorname{lc}(U) U, \mathrm{pp}\left(H^{*}\right)$ is a factor of $U$ over $\mathbf{Z}$.

Because $U$ is squarefree, different irreducible factors of $U$ over the integers cannot involve the same $P_{i}$ for any $i$. Exactly how these $P_{i}$ 's should be grouped to give rise to the irreducible factors of $U$ requires a combinatorial search.

With $U, h, P_{i}, i=1,2, \ldots, r$, as input, the following algorithm returns a list of irreducible factors of $U$ over $\mathbf{Z}$. This algorithm finds the true factors by trial division over $\mathbf{Z}$. The divisors are formed by taking different products, $\bmod \left(b, b^{h}\right)$, of 1 , $2, \ldots, r-2$ elements, in that order, from the set $\left\{P_{1}, P_{2}, \ldots, P_{r}\right\}$. Of all such products, only those of degree not exceeding $m / 2$ are formed. If $U$ is not monic, necessary steps for correcting the leading coefficients of possible factors as indicated in (20) will be taken. Each successful division produces an irreducible factor of $U$ and replaces $U$ by the quotient. This algorithm ends when $U=1$ or when all combinations are taken. In the latter case, the remaining quotient $U$ is an irreducible factor.

\section{Algorithm TRUEFACTORS:}

(1) For $i=1$ through $r$, execute the following:

Set $U^{*}=\operatorname{lc}(U) U, Y=\operatorname{lc}(U) \operatorname{lc}\left(P_{i}\right)^{-1} P_{i} \bmod \left(b, \xi^{h}\right)$. If $Y$

divides $U^{*}$ over $\mathrm{Z}$, put $\mathrm{pp}(Y)$ on the list FAC and set

$U=U / \mathrm{pp}(Y)$; otherwise put $P_{i}$ on the list $L$.

(2) If $L$ is an empty list, return the answer FAC and exit. If $L$ contains less than four elements, put $U$ on the list FAC return

FAC and exit (for $L$ cannot contain two disjoint subsets of two

or more elements). Otherwise, set $M=1, \alpha=$ number of elements on $L$,

$r=r-$ number of elements on FAC, $u=\operatorname{deg}(U) / 2, U^{*}=\operatorname{lc}(U) U$.

(3) Set $M=M+1$.

(4) If $U=1$, return FAC and exit. If $r=1, M \geqslant r-1$ or $M>u$, put $U$ on the list FAC, return FAC and exit.

(5) Select a combination, $E$, of $M$ elements from $L$ with the sum of their degrees not to exceed $u$. If no such combination can be found, put $U$ on the list FAC, return FAC and exit. If all such combinations of $M$ elements from $L$ have been taken, go to step 3 .

(6) Set $Y=$ product of elements in $E \bmod \left(b, b^{h}\right)$.

Set $Y=\operatorname{lc}(U) \operatorname{lc}(Y)^{-1} Y \bmod \left(b, b^{h}\right)$. If $Y$ divides $U^{*}$ over $\mathrm{Z}$, do the following:

(i) Put $\mathrm{pp}(Y)$ on the list FAC and set $L=L-E, r=r-1$;

(ii) set $U=U / \mathrm{pp}(Y), u=\operatorname{deg}(U) / 2, U=\operatorname{lc}(\operatorname{pp}(Y)) U$;

(iii) delete from $L$ any element with degree greater than $u$; set $\alpha=$ number of elements on $L$.

(iv) go to step 4.

If $Y$ does not divide $U^{*}$ over $\mathbf{Z}$, go to step 5 .

In the above algorithm, the computing of $U^{*}$ and $\operatorname{pp}(Y)$ is unnecessary if the original polynomial $U$ is monic. Trial division of $U^{*}$ by $Y$ is preceded by test divisions of the leading and trailing coefficients. 
Note that only one multiplication is needed to form a product of $M$ elements from that of $M-1$ elements. In the worst case, where $U$ is irreducible and $r=$ $\operatorname{deg}(U)=m$, this algorithm goes through $2^{m-1}$ iterations and each iteration involves essentially one multiplication and one division of multivariate polynomials.

10. Special Cases. The factoring process for polynomials of some particular forms can be greatly speeded up by the use of special techniques. For instance, the factors of $x^{n} \pm 1$ can be obtained by computing the factors of the relevant cyclotomic polynomials [10]. For other univariate polynomials, Eisenstein's irreducibility criterion [10] can be applied first. In addition to these, we have in MACSYMA the following special cases that have been implemented by R. Fateman:

A. Linear Case: If $U$ is a polynomial linear in the variable $x$, i.e., $U=a x+b$, then $U$ is irreducible if and only if $\operatorname{GCD}(a, b)=1$.

B. Quadratic Case. If $U$ is quadratic in some variable $x$, that is, $U=a x^{2}+b x+$ $c$, and $U$ is primitive with respect to $x$. Then, either $U$ is irreducible over the integers or factors into two linear factors computed by the quadratic formula.

11. Practical Difficulties. In early tests with the factoring program, it was discovered that taking GCD of multivariate polynomials was very slow. This affected the very first step in our factoring algorithm. Namely, content and squarefree operations were taking too long. At that time, MACSYMA was using the modular GCD algorithm [3]. The need for a better method for multivariate GCD motivated the work on the EZ-GCD algorithm [8], [11]. The EZ-GCD algorithm uses the extended Zassenhaus algorithm. It is very efficient for multivariate polynomials that are not completely dense. It also makes content taking extremely fast.

There are still two major sources of difficulty. One is the coefficient bound which we have mentioned in Section 6. The other has to do with being able to use zeros for the $a_{i}$ 's. An important step in the extended Zassenhaus algorithm is the change of variable

$$
V\left(x, y_{2}, \ldots, y_{n}\right)=U\left(x, y_{2}+a_{2}, \ldots, y_{n}+a_{n}\right) .
$$

If not enough of the $a_{i}$ 's are zero, one can expect $V$ to have many more terms than $U$. Just compare the size of $x^{r} y^{s} z^{t}$ with $(x+1)^{r}(y+2)^{s}(z+3)^{t}$. The former has only one term and the latter has $(r+1)(s+1)(t+1)$ terms when expanded. This intermediate expression growth is typical of many problems in symbolic manipulation. One may try avoiding this change of variable. But this is not always easily done. We have yet no general solution for this growth problem.

Appendix. This appendix contains 18 examples of factoring done by the MACSYMA system (version 252) at Project MAC, M.I.T. The running times are indicated in milliseconds. We have chosen the examples with the following complexity measurements of polynomials relative to factorization in mind: degree, number of variables, density, number of irreducible factors, number of terms in the polynomial, size and form of the coefficients, especially the leading coefficient. For each example, the or- 
der of the variables is $X, Y, Z, W$, with $X$ the most main variable and $W$ the least main. In MACSYMA, labels (Ci) and (Di) are used for the $i$ th command and display lines respectively. The symbol \% stands for the previous expression.

(D1) $\quad x^{14}-1$

(D2) $(x-1)(x+1)\left(x^{6}+x^{5}+x^{4}+x^{3}+x^{2}+x+1\right)\left(x^{6}-x^{5}+x^{4}-x^{3}+x^{2}-x+1\right)$

(D) FACTOR (s): $\quad x^{8}+x+1$

TIMB = 1005 MSEC.

(D4)

$$
\left(x^{2}+x+1\right)\left(x^{6}-x^{5}+x^{3}-x^{2}+1\right)
$$

(D5) $228533760 x^{6}+1921081160 x^{5}+233096077 x^{4}-204462708 x^{3}+170301571 x^{2}=291338682 x+7552512$ (D) FACTOR(א):

(D6)

(D7) $\operatorname{FACTOR}(\$)$;

TIME $=526$ MSEC.

(DB)

(D9) FACTOR (8);

TIIDE $=421 \mathrm{FACPEC}$.

(D10)

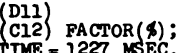

(D12)

(D13) $\mathrm{DACTOR}($ () $)$ :

TINE $=908$ MSEC;

(D14)

$$
\left(455 x^{2}+3750 x-99\right)\left(576 x^{2}+131 x-256\right)\left(872 x^{2}-55 x+298\right)
$$

$$
x^{3}-y^{3}
$$

$$
\begin{aligned}
& x^{4}+y^{3}+z^{5} \\
& z^{5}+y^{3}+x^{4}
\end{aligned}
$$$$
(X-Y)\left(Y^{2}+X Y+X^{2}\right)
$$

$$
2 x^{4}+\left(y^{2}+10 z^{2}\right) x^{2}-6 y^{4}-z^{2} y^{2}+12 z^{4}
$$

(D15) FACTOR(\$);

TIME $=2309$ MSEC.

(D16)

$$
\left(3 z^{2}+2 y^{2}+x^{2}\right)\left(4 z^{2}-3 y^{2}+2 x^{2}\right)
$$

$$
y^{3} x^{3}+\left(2 z Y^{2}+9 y\right) x^{2}+\left(z Y^{3}+z^{2} Y+9 z\right) x+z^{2} Y^{2}+9 z Y-
$$

$$
\text { (D17) } \begin{aligned}
x^{4}+(-z+3) x^{3}+\left(-y^{2}+z y+z^{3}-3 z-13\right) x^{2}+\left(z y^{2}+3 z y-z^{4}\right. & +15 z+6) x-z y^{3}-2 y^{2} \\
& +\left(z^{4}-15 z\right) y+2 z^{3}-30
\end{aligned}
$$

(C18) PLCTOR(\&);

(D18)

$$
\left(y z-x z+x^{2}+2\right)\left(z^{3}-y^{2}+x^{2}+3 x-15\right)
$$

(D19) $\operatorname{PACTOR}(8)$;

TIME $=1025$ MSEC.

$$
x^{6}+y^{6}
$$

(D20)

$$
\left(Y^{2}+x^{2}\right)\left(Y^{4}-x^{2} Y^{2}+x^{4}\right)
$$

(D21)

$$
\begin{aligned}
2115 x^{4}+\left(45 w^{2} z^{3}-45 w^{2}\right) x^{3}+\left(-470 Y^{3}+\left(141 z^{3}+94 w z\right) Y\right) x & +\left(-10 w^{2} z^{3}+10 w^{2}\right) Y^{2} \\
& +3 w^{2} z^{6}+2 w^{3} z^{4}-3 w^{2} z^{3}-2 w^{3} z
\end{aligned}
$$

(C22) FACTOR(8)

$$
\begin{aligned}
& \text { (D22) }\left(3 z^{3}+2 w z-10 Y^{2}+45 x^{3}\right)\left(w^{2} z^{3}+47 x y-w^{2}\right)
\end{aligned}
$$

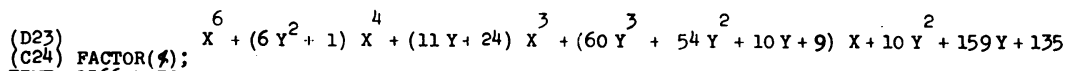


(D24)

$$
\left(10 Y+X^{3}+9\right)\left(X\left(6 Y^{2}+1\right)+Y+X^{3}+15\right)
$$

(D25)

$$
\begin{array}{r}
\left((z-w) y^{2}+\left(-z^{2}+w^{2}\right) y+w^{2}-w^{2} z\right) x^{3}+\left((-z+w) y^{3}+\left(z^{3}-w^{3}\right) y-w^{3}+w^{3} z\right) x^{2} \\
+\left(\left(z^{2}-w^{2}\right) y^{3}+\left(-z^{3}+w^{3}\right) y^{2}+w^{2} z^{3}-w^{3} z^{2}\right) x+\left(-w z^{2}+w^{2} z\right) y^{3}+\left(w^{3}-w^{3} z\right) y^{2} \\
+\left(-w^{2} z^{3}+w^{3} z^{2}\right) y
\end{array}
$$

(C26) FACTOR(\$);

(D26)

$$
(\mathrm{X}-\mathrm{W})(\mathrm{Y}-\mathrm{W})(\mathrm{X}-\mathrm{Y})(\mathrm{Z}-\mathrm{W})(\mathrm{X}-\mathrm{Z})(\mathrm{Y}-\mathrm{Z})
$$

$$
\left(Y^{3}+Z Y^{2}+Z^{2} Y+Z^{3}\right) X^{5}+\left(Y^{2}+(Z+90) Y+90 Z\right) X^{3}+\left((Z-11) Y^{2}+Z^{3}-11 Z^{2}\right) X^{2}+(Z-11) Y
$$

(C28) FACTOR (4);

TIME $=5072$ MSEC.

(D28)

$$
\begin{aligned}
&\left(X^{3}(Y+Z)+Z-11\right)\left(X^{2}\left(Z^{2}+Y^{2}\right)+Y+90\right) \\
& Z Y X^{3}+\left(\left(Z^{2}+1\right) Y^{2}+(20 Z+30) Y+Z^{2}+10 Z\right) X^{2} \\
&+\left(Z Y^{3}+(30 Z+20) Y^{2}+\left(Z^{3}+10 Z^{2}+Z+610\right) Y+20 Z^{2}+230 Z+300\right) X+\left(Z^{2}+10 Z\right) Y^{2} \\
&+\left(30 Z^{2}+320 Z+200\right) Y+600 Z+6000
\end{aligned}
$$

(C30) FACTOR( (\$)

TIME $=4670 \mathrm{MSEC}$

$(Z+X Y+10)(X Z+Y+30)(Y Z+X+20)$

(D31)

$$
\begin{aligned}
& \left(54 z^{2} Y^{6}-216 w^{2} z^{5} Y^{5}+522 z^{2} Y^{4}\right) x^{10}+\left(18 w^{2} z^{5}-18 w^{2} z^{2}\right) y^{4} x^{9} \\
+ & \left(-24 z^{2} Y^{7}+96 w^{2} z^{5} Y^{6}-232 z^{2} Y^{5}\right) x^{8}+\left(\left(42 w^{2}-3\right) z^{2} Y^{7}+\left(-168 w^{4}+12 w^{2}\right) z^{5} Y^{6}\right. \\
+ & \left.\left(\left(-8 w^{2}+9\right) z^{5}+6 w z^{3}+\left(414 w^{2}-29\right) z^{2}\right) Y^{5}+\left(-36 w^{2} z^{8}-24 w^{3} z^{6}\right) Y^{4}+\left(87 z^{5}+58 z^{3}\right) Y^{3}\right) x^{7} \\
+ & \left(\left(\left(14 w^{4}-w^{2}\right) z^{5}+\left(-14 w^{4}+w^{2}\right) z^{2}\right) Y^{5}+\left(3 w^{2} z^{8}+2 w^{3} z^{6}-3 w^{2} z^{5}-2 w^{3} z^{3}\right) Y^{3}\right) x^{6}
\end{aligned}
$$

(C32) $\operatorname{FACTOR}(4)$;

(D32) $X^{6} Y^{3} Z^{2}\left(3 Z^{3}+2 W Z-8 X Y^{2}+\left(14 W^{2}-1\right) Y^{2}+18 X^{3} Y\right)\left(X\left(-12 W^{2} Y Z^{3}+3 Y^{2}+29\right)+W^{2} Z^{3}-W^{2}\right)$

$$
6 Y^{4} X^{8}+35 Y^{4} X^{7}+\left(75 Y^{4}+53 Y^{3}\right) X^{6}+\left(70 Y^{4}+226 Y^{3}\right) X^{5}+\left(24 Y^{4}+314 Y^{3}+169 Y^{2}\right) X^{4}
$$

(C34) FACTOR(\$);

$$
+\left(142 Y^{3}+465 Y^{2}\right) X^{3}+\left(311 Y^{2}+227 Y\right) X^{2}+298 Y X+105
$$

$$
\begin{aligned}
& (D 34)=16374 \text { MSEC. }\left(X^{2} Y+X Y+1\right)\left(X^{2} Y+2 X Y+3\right)\left(2 X^{2} Y+3 X Y+5\right)\left(3 X^{2} Y+4 X Y+7\right) \\
& \text { (D35) } Y^{2} X^{6}+\left(Z Y^{3}+Z^{4}+1\right) X^{4}+\left(Y^{4}+Z^{3} Y^{2}+Z\right) X^{3}+\left(Z^{5}+Z\right) Y X^{2}+\left(\left(Z^{4}+1\right) Y^{2}+Z^{2} Y+Z^{7}+Z^{3}\right) X+Z Y^{2}+Z^{4} \\
& \text { (C36) } \operatorname{FACTOR}(\text { (x) : } \\
& \text { (D36) } \quad\left(z^{3}+X Y z+y^{2}+x^{3}\right)\left(X\left(z^{4}+1\right)+z+x^{3} y^{2}\right)
\end{aligned}
$$

Project MAC and Department of Mathematics

Massachusets Institute of Technology

Cambridge, Massachusetts 02139

School of Mathematics

Institute for Advanced Studies

Princeton, New Jersey 08540

1. E. R. BERLEKAMP, "Factoring polynomials over finite fields," Bell System Tech. J., v. 46, 1967, pp. 1853-1859. MR 36 \#2314.

2. E. R. BERLEKAMP, "Factoring polynomials over large finite fields," Math. Comp., v. 24, 1970, pp. 713-735. MR 43 \# 1948.

3. W. S. BROWN, "On Euclid's algorithm and the computation of polynomial greatest common divisors," J. Assoc. Comput. Mach., v. 18, 1971, pp. 478-504. MR 46 \#6570.

4. G. E. COLLINS, SAC-1 Modular Arithmetic System, University of Wisconsin Technical Report No. 10, June 1969.

5. A. O. GEL'FOND, Transcendental and Algebraic Numbers, GITTL, Moscow, 1952; English transl., Dover, New York, 1960. MR 15, 292; 22 \#2598.

6. D. E. KNUTH, The Art of Computer Programming. Vol. 2: Seminumerical Algorithms, Addison-Wesley, Reading, Mass., 1969. MR 44 \#3531. 

1963.

7. J. McCARTHY et al., LISP 1.5 Programmer's Manual, M.I.T. Press, Cambridge, Mass.,

8. J. MOSES \& D. Y. Y. YUN, "The EZ GCD algorithm," Proceedings of ACM Annual Conference, August 1973.

9. D. R. MUSSER, Algorithms for Polynomial Factorization, Ph. D. Thesis, Computer Science Department, The University of Wisconsin, Madison, Wis., 1971.

10. B. L. VAN DER WAERDEN, Modern Algebra. Vol. 1, Springer, Berlin, 1930; English transl., Ungar, New York, 1949. MR 10, 587.

11. D. Y. Y. YUN, The Hensel Lemma in Algebraic Manipulation, Ph. D. Thesis, Department of Mathematics, M.I.T., Nov. 1973 (also Project MAC TR-138, November 1974).

12. H. ZASSENHAUS, “On Hensel factorization. I," J. Number Theory, v. 1, 1969, pp. 291 311. MR $39 \# 4120$.

13. MACSYMA Reference Manual, the MATHLAB group, Project MAC, M.I.T., Cambridge, Mass., Sept. 1974. 